„DIE LEISTUNGEN DER STUDENTENWERKE SIND EIN ERFOLGSFAKTOR

FÜR DEN BOLOGNA-PROZESS, FÜR STUDIENZUGANG UND STUDIEN-

ERFOLG. DOCH ES MUSS FAST SCHON VON EINEM RÜCKZUG

DES STAATES AUS DER SOZIALEN VERANTWORTUNG FÜR SEINE

STUDIERENDEN GESPROCHEN WERDEN.“

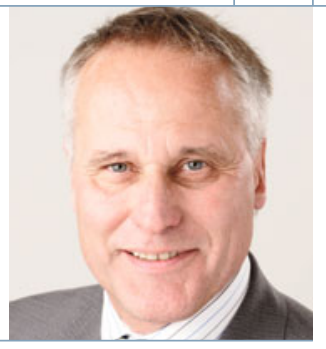

Achim Meyer auf der Heyde

\title{
Mehr als Mensa und BAföG - Studentenwerke stärken
}

Das deutsche Hochschulsystem wähnt sich für die Rekordzahlen von Erstsemestern und die insgesamt rund 2,4 Millionen Studierenden gerüstet. Stehen denn nicht über die gemeinsamen Hochschulpakte von Bund und Ländern ausreichend Studienplätze zur Verfügung, um das Studierendenhoch aufzufangen? Auf den ersten Blick könnte man antworten: Ja. Auf den zweiten Blick sieht die Sache jedoch anders aus.

Was nämlich gerne vergessen wird: Ein Kalkulieren in Studienplätzen allein reicht nicht aus. Es mag banal klingen, aber die Studierenden benötigen nicht nur einen Studienplatz - sie müssen günstig unterkommen, hochschulnah und preiswert essen, ihre Studienfinanzierung muss stehen, sie benötigen Beratung, vielleicht Kinderbetreuung. Um ein berühmtes Wort von Max Frisch abzuwandeln: „Wir suchten Fachkräfte, und es kommen Menschen." Immer mehr solcher Menschen, immer mehr junge Menschen, mit ganz menschlichen Bedürfnissen.

Hier kommen die Studentenwerke ins Spiel. Formal unabhängig von den Hochschulen, aber mit ihnen in enger Kooperation verbunden, fördern die 58 Studentenwerke in Deutschland die 2,4 Millionen Studierenden „wirtschaftlich, sozial, gesundheitlich und kulturell“. So heißt es in den entsprechenden Ländergesetzen, denen die Studentenwerke als Anstalten des öffentlichen Rechts verpflichtet sind.

Konkret: Die 58 Studentenwerke betreiben an den Hochschulen 823 Mensen und Cafeterien, bieten 179.255 Plätze in mehr als 1.000 Wohnheimen, 7.345 Plätze in 221 Kindertagesstätten. Jährlich zahlen die Studentenwerke im Auftrag von Bund und Ländern 1,5 Milliarden Euro BAföG an etwa ein Viertel der Studierenden. Die meisten Studentenwerke bieten auch psychologische Beratung mit psychologischem und sozialem Schwerpunkt sowie für Studierende mit Behinderung oder chronischer Krankheit.

Die Arbeitsteilung im deutschen Hochschulsystem hat sich bewährt. Die Hochschulen bespielen die Bühnen Forschung und Lehre, die Studentenwerke die Bühne des studentischen Lebens.

Aber zwischen Hochschulen und Studentenwerken gibt es einen entscheidenden Unterschied in der Finanzierungsstruktur: Während die Hochschulen gänzlich staatlich finanziert werden, erwirtschaften die Studentenwerke inzwischen zwei Drittel ihrer Einnahmen selbst. Der Zuschuss der Länder, also der staatliche Finanzierungsanteil, sinkt kontinuierlich. Trugen die Landeszuschüsse 1992 noch zu fast einem Viertel der Gesamtfinanzierung der Studentenwerke bei, so sind sie inzwischen auf einen Tiefstand von zehn Prozent gefallen. Die sinkenden Zuschüsse konnten nur zum Teil durch die Steigerung der Semesterbeiträge der Studierenden von 9,7 Prozent im Jahr 1992 auf 14,6 Prozent im Jahr 2010 kompensiert werden.

Diese Negativentwicklung bei den Länderzuschüssen steht im Gegensatz zur politischen Rhetorik. Die Kultusministerkonferenz hatte noch im Oktober 2009 die Bedeutung der Service- und Beratungsangebote für Studierende anerkannt und gar eine Stärkung der Studentenwerke für notwendig erklärt. Passiert ist nichts.

Weiterhin wird der Ausbau der Studentenwerke parallel zu den zusätzlichen Plätzen im Hochschulpakt nicht gefördert, obwohl Studieren ohne die notwendige soziale Infrastruktur kaum gelingt. Die 19. Sozialerhebung des Deutschen Studentenwerks zur sozialen Lage der Bachelor-Studierenden zeigt: Bachelor-Studierende wohnen tendenziell häufiger im Wohnheim, sie gehen häufiger in die Studentenwerks-Mensa, sie beziehen häufiger BAföG, und sie haben einen höheren Beratungsbedarf als ihre Kommilitonen in anderen
Studiengängen. Kurz: Die Leistungen der Studentenwerke sind ein Erfolgsfaktor für den Bologna-Prozess, für Studienzugang und Studienerfolg.

Als Teil der staatlichen Daseinsvorsorge erfüllen die Studentenwerke mit ihren Leistungen öffentliche Aufgaben. Die dafür bereitgestellten öffentlichen Finanzmittel sind in einem Ausmaß zurückgegangen, dass fast schon von einem Rückzug des Staates - vor allem der Länder - aus der sozialen Verantwortung für deren Studierenden gesprochen werden muss.

Ich bin überzeugt: Nur mit zusätzlichen Investitionen in die soziale Infrastruktur des Studiums, parallel zur Schaffung zusätzlicher Studienplätze, kann der Studienerfolg der vielen zusätzlichen Studierenden, die erfreulicherweise an unsere Hochschulen kommen, nachhaltig gesichert werden.

Hier sehe ich den Bund und die Länder gemeinsam in der Pflicht. Warum sollte bei der sozialen Infrastruktur nicht möglich sein, was bei den Hochschulpakten als gemeinsame Bund-Länder-Programme möglich ist?

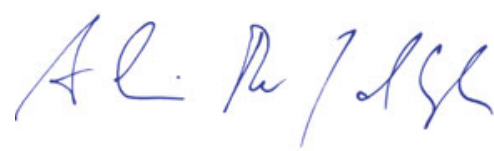

Achim Meyer auf der Heyde,

Generalsekretär Deutsches Studentenwerk, Berlin

Korrespondenzadresse:

Achim Meyer auf der Heyde

Deutsches Studentenwerk

Monbijouplatz 11

D-10178 Berlin

Tel.: 030-297727-12

Fax: 030-297727-99

achim.meyeraufderheyde@studentenwerke.de 